\title{
Topical cyclosporin as an alternative treatment for vision threatening blepharokeratoconjunctivitis: a case report
}

\author{
Abdul-Salim Ismail ${ }^{1,2}$ \\ Rohana Taharin ${ }^{2}$ \\ Zunaina Embong' \\ 'Department of Ophthalmology, \\ School of Medical Sciences, \\ Universiti Sains Malaysia, Kubang \\ Kerian, Kelantan, ${ }^{2}$ Department \\ of Ophthalmology, Hospital \\ Pulau Pinang, Jalan Resindensi, \\ Pulau Pinang, Malaysia
}

This article was published in the following Dove Press journal:

International Medical Case Reports Journal

26 June 2012

Number of times this article has been viewed
Correspondence: Zunaina Embong Department of Ophthalmology, School of Medical Sciences, Universiti Sains Malaysia, 16150 Kubang Kerian, Kelantan, Malaysia

Tel +6097676362

Fax +6097673370

Email zunaina@kb.usm.my

\begin{abstract}
Here, a case of vision threatening blepharokeratoconjunctivitis that responded well to topical cyclosporin is reported. A 9-year-old Malay girl with a history of bilateral blepharokeratoconjunctivitis was regularly treated with lid scrubbing using diluted baby shampoo, fusidic acid gel, and topical steroids as well as an intermittent course of oral doxycycline for the past year. She developed acute onset bilateral eye redness associated with poor vision in her right eye. Both eyes showed marked diffuse hyperemic conjunctiva with corneal vascularization. The presence of corneal vascularization obscured the visual axis in the right eye. The condition did not improve with regular intensive lid hygiene using diluted baby shampoo, fusidic acid gel, and topical steroids. She was started on topical cyclosporin A $0.5 \%$ every 6 hours. There was a dramatic regression of corneal vascularization after 3 days on topical cyclosporin, with marked improvement in visual acuity. This is a single case in which cyclosporin improved the status of the ocular surface. A large cohort study is required to justify its effectiveness in treating blepharokeratoconjunctivitis and to test its potential as an alternative immunosuppressive agent in comparison to conventional corticosteroids.
\end{abstract}

Keywords: blepharokeratoconjunctivitis, cyclosporin

\section{Introduction}

Blepharokeratoconjunctivitis is a condition that is defined clinically as the presence of eyelid involvement by lid margin scarring, blepharitis, meibomitis, and chalazion or hordeolum, accompanied by epithelial or stromal keratitis, corneal scarring, vascularization, and thinning or perforation. ${ }^{1}$ It is a common condition in children, with the mean age at presentation around 10 years. ${ }^{1}$ Children with blepharokeratoconjunctivitis often present with symptoms of eye redness $(68.6 \%),{ }^{1}$ photophobia $(63.6 \%),{ }^{2}$ tearing $(50.0 \%),{ }^{2}$ pain $(21.6 \%),{ }^{1}$ and irritation $(13.7 \%) .{ }^{1}$ Common clinical signs include corneal scarring $(92.2 \%)$, superficial corneal vascularization $(90.2 \%)$, meibomitis $(86.3 \%)$, blepharitis $(76.5 \%)$, punctate epithelial erosions or superficial punctate keratitis (74.5\%), conjunctival hyperemia (51.0\%), and chalazion $(45.1 \%){ }^{1}$ Blepharokeratoconjunctivitis has a chronic course characterized by exacerbation and remissions. Recurrent and prolonged inflammation may lead to severe permanent ocular surface damage.

The treatment for pediatric blepharokeratoconjunctivitis includes eyelid hygiene, warm compresses, and a combination of topical and systemic antibiotics. ${ }^{3}$ During acute exacerbations, a topical corticosteroid has been used to control conjunctival and corneal inflammation. ${ }^{2}$ 
Blepharokeratoconjunctivitis is the result of an underlying cytokine and inflammatory-mediated process affecting both the meibomian glands and the ocular surface. ${ }^{4}$ Topical corticosteroids inhibit proinflammatory cytokines by inhibiting transcriptase factors (eg, nuclear factor- $\kappa \mathrm{B}$ ). However, their long-term use is known to cause side effects such as reactivation of infectious keratitis, glaucoma, and cataracts.

Cyclosporin is an established immune modulating drug that was originally used to prevent graft rejection. Topical preparation became commercially available in recent years for usage in ocular surface-related diseases. ${ }^{5}$ Cyclosporin is a cyclic polypeptide produced as a metabolite by the fungus Tolypocladium inflatum. ${ }^{6}$ It inhibits the activity of transcriptase factors of the nuclear factor of activated $T$ cells, interfering with the induction of cytokines and other inducible genes required for the immune response. ${ }^{6}$ Fukushima et al demonstrated in an animal model that immune-mediated blepharoconjunctivitis is T cell-mediated, ${ }^{7}$ and staphylococcus toxins may be the causative agent in humans. ${ }^{8}$ Besides that, cyclosporin also showed better meibomian gland secretion and stable tear film compared to a conventional corticosteroid. ${ }^{4}$ Cyclosporin has been reported to be helpful as a primary or adjunctive therapy in the treatment of dry eye, ${ }^{9}$ Thygeson's superficial punctate keratitis, ${ }^{10}$ superior limbic keratoconjunctivitis, ${ }^{11}$ and meibomian gland dysfunction. ${ }^{12}$

Here, a case of acute exacerbation of vision threatening bilateral blepharokeratoconjunctivitis in a young girl is reported. She did not respond to intensive treatment and topical corticosteroids; however, responded well to topical cyclosporin without any side effects.

\section{Case report}

A 9-year-old Malay girl was diagnosed to have bilateral blepharokeratoconjunctivitis with intermittent exacerbation and was under the authors' care for the past year. She was being treated with regular lid scrubbing using diluted baby shampoo, fusidic acid gel, and topical dexamethasone $0.1 \%$ as well as an intermittent course of oral doxycycline $50 \mathrm{mg}$ daily for 4-6 weeks. Her symptoms usually resolved after each course of intensive treatment, but the condition tended to exacerbate frequently.

She developed acute onset of bilateral eye redness associated with poor vision in her right eye. Visual acuity in her right eye deteriorated from 6/9 to light perception. However, visual acuity in her left eye remained at 6/12. Both eyes showed marked diffuse conjunctival hyperemia. The presence of corneal vascularization obscured the visual axis in the right eye, with old central corneal scarring (Figure 1). In the left eye, there was also presence of peripheral corneal vascularization with old central corneal scarring (Figure 1). The lid margin showed features of meibomian gland dysfunction with a presence of posterior lid margin telangiectasia, inflammation, and capping of the meibomian glands in both eyes.

Staphylococcal aureus was isolated from her conjunctival and lid swab, which was sensitive to fusidic acid. She was treated with intensive lid hygiene using diluted baby shampoo, fusidic acid gel every 8 hours, and topical dexamethasone $0.1 \%$ every 6 hours for both eyes.

Her right eye condition remained the same despite intensive treatments. A trial with topical cyclosporin A $0.5 \%$ every 6 hours was decided on, which was the authors' first experience using topical cyclosporin for blepharokeratoconjunctivitis. There was a dramatic regression of corneal vascularization in both eyes after 3 days on topical cyclosporin (Figure 2). After 1 week of treatment, both eyes showed white conjunctiva with resolved corneal vascularization (Figure 3 ). Her visual acuity was markedly
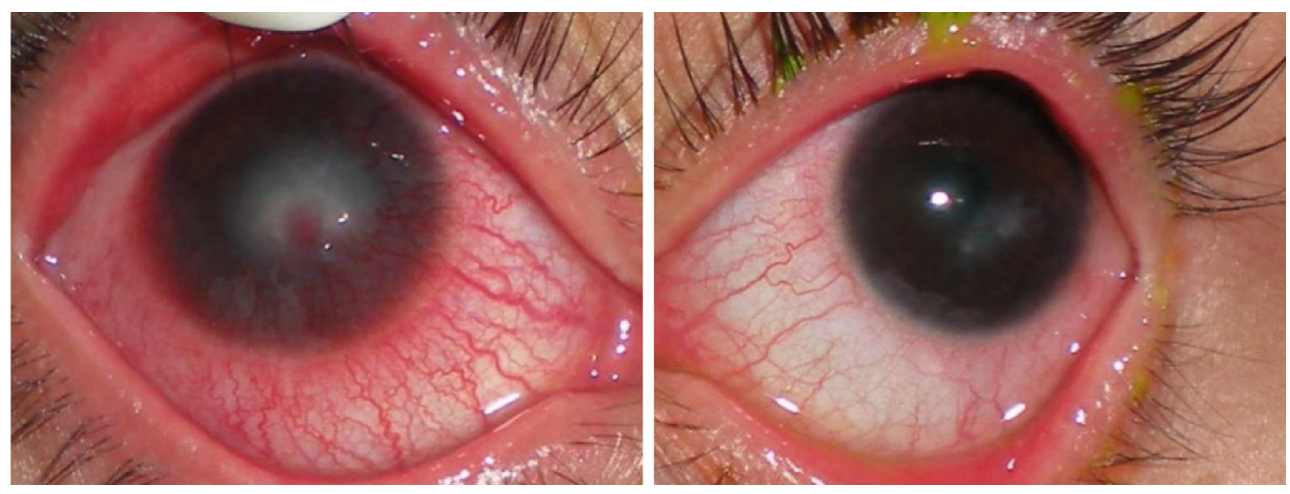

Figure I Corneal vascularization of the right eye (left photo) and left eye (right photo) before treatment with topical cyclosporin. 

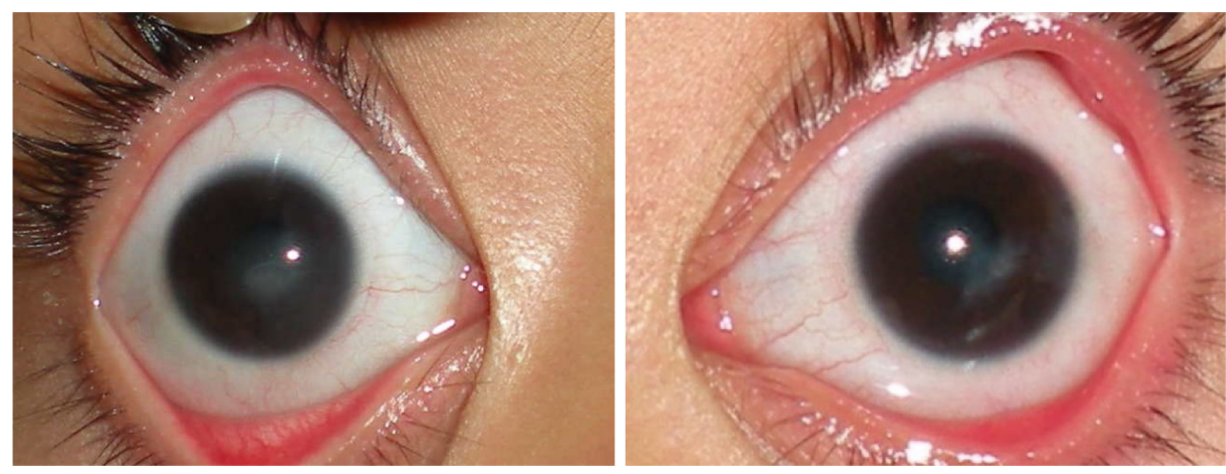

Figure 2 Dramatic regression of corneal vascularization in both eyes (right eye: left photo, left eye: right photo) after 3 days on topical cyclosporin.

improved to $6 / 9$ for both eyes. Her lid margin appeared clean and healthy with no meibomian capping. Her tear film also seemed to be more stable with improved tear break-up time.

At 1-month follow-up, her ocular surface looked stable and healthy. The frequency of topical cyclosporin was reduced from every 6 hours to every 12 hours; however, after 2 weeks on topical cyclosporin A $0.5 \%$ every 12 hours, there was a reappearance of conjunctival hyperemia and corneal vascularization. In view of this recurrence, it was decided that the frequency of topical cyclosporin A $0.5 \%$ every 6 hours would be maintained for a longer period. Every attempt to taper cyclosporin treatment resulted in a reappearance of symptoms and signs of blepharokeratoconjunctivitis. It was decided that topical cyclosporin A $0.5 \%$ every 6 hours would be kept for up to 6 months before attempting to taper down the frequency. During this period, the patient was closely monitored for local side effects. There was no ocular burning, eye stinging, nor conjunctival hyperemia noted during follow-up. Follow-up at 6 months after cessation of treatment showed that there was no recurrence of the disease and the ocular surface was stable and healthy.

\section{Discussion}

Treatment for blepharokeratoconjunctivitis is the same as treatment for posterior blepharitis or meibomian gland dysfunction, ${ }^{13}$ which includes eyelid hygiene, warm compresses, and a combination of topical and systemic antibiotics. ${ }^{3}$ Daily lid hygiene removes debris and encrustations along the lid margin using cotton wool-tipped swab sticks with diluted baby shampoo, warm water, diluted bicarbonate solution, ${ }^{2}$ or special eyelid cleansers. ${ }^{3}$ In the current case report, the patient was being treated with regular lid scrubbing using diluted baby shampoo.

Common organisms isolated from conjunctival or lid swabs in patient with blepharokeratoconjunctivitis are $S$. aureus, ${ }^{2,3,14}$ S. epidermidis, ${ }^{2,3}$ and Propionibacterium acnes. ${ }^{14} \mathrm{~A}$ variety of topical antibiotics has been used to reduce the bacterial load, including chloramphenicol, ${ }^{3}$ fusidic acid gel, ${ }^{1,3}$ levofloxacin, ${ }^{1}$ tobramycin, ${ }^{1}$ erythromycin, ${ }^{3}$ ciprofloxacin, ${ }^{3}$ and gentamicin. ${ }^{3}$ Systemic antibiotics are often necessary to control the lid disease. Oral erythromycin is an effective systemic antibiotic and tetracycline is contraindicated in children younger than 8 years because of its effect on dental enamel. ${ }^{3}$ In a study done by Teo et al,
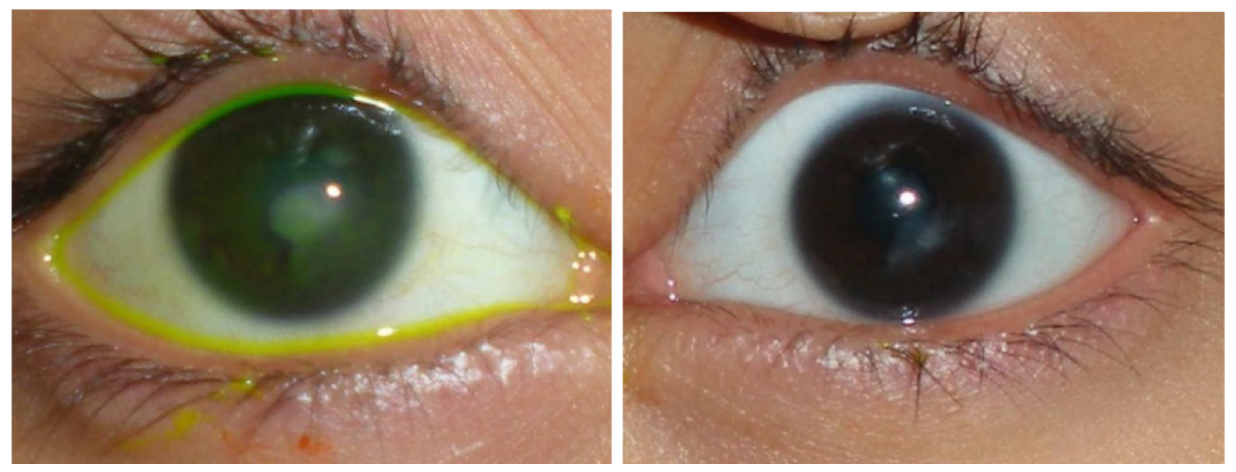

Figure 3 Resolved corneal vascularization in both eyes (right eye: left photo, left eye: right photo) after I week on topical cyclosporin. 
children younger than 9 years of age were treated with oral erythromycin or Augmentin ${ }^{\circledR}$, whereas older children were treated with oral doxycycline. ${ }^{1}$ The patient in the current case report was treated with an intermittent course of oral doxycycline because she is in the older age group. She also had complete primary dentition when the treatment was initiated. Fusidic acid gel was prescribed based on swab culture and sensitivity.

Topical corticosteroid was used to control the conjunctival and corneal inflammation. Although corticosteroids are widely used as a primary ocular immunosuppressive agent, long-term use can induce various vision threatening side effects including glaucoma, cataracts, ulcers, and reactivation of viral keratitis. ${ }^{15}$ In the current patient, topical cyclosporin was introduced after an adequate trial of intensive treatments including steroids, which failed to show any improvement in the patient's condition. There was dramatic regression of the ocular surface inflammation with improved vision after initiation of topical cyclosporin.

Cyclosporin specifically interferes with the underlying mechanism in meibomian gland dysfunction by targeting T cells. ${ }^{6}$ Cyclosporin has advantages over corticosteroids, since there is no suppression of normal host immune response and enhancement of collagenolytic enzyme production with subsequent corneal thinning. A study by Rubin and Rao showed that patients treated with cyclosporin for 12 weeks had increased meibomian gland secretion, a stable tear film, and a high mean secretion quality, with improvements in symptoms of blurred vision, itching, burning, lid erythema, and telangiectasia. ${ }^{4}$ Ocular burning is the most common adverse event associated with the ophthalmic use of cyclosporin. ${ }^{9}$ No drug-related adverse events occurred in the patient in the current case report.

Many ophthalmologists worldwide still use corticosteroids as the main treatment for blepharokeratoconjunctivitis. Based on this single case, results are promising but a full investigation with a large population would be required to change conventional clinical practice in terms of using cyclosporin as a first-line treatment to prevent permanent ocular surface damage in blepharokeratoconjunctivitis.

\section{Conclusion}

This is a single case in which cyclosporin improved the status of the ocular surface. A large cohort study is required to justify its effectiveness in treating blepharokeratoconjunctivitis and to test its potential as an alternative immunosuppressive agent in comparison to conventional corticosteroids.

\section{Acknowledgment}

The authors would like to thank Universiti Sains Malaysia for providing the financial support for this case report (Research University Grant: 1001/PPSP/812064).

\section{Disclosure}

The authors report no conflicts of interest in this work.

\section{References}

1. Teo L, Mehta JS, Htoon HM, Tan DT. Severity of pediatric blepharokeratoconjunctivitis in Asian eyes. Am J Ophthalmol. 2012; 153(3):564-570.

2. Viswalingam M, Rauz S, Morlet N, Dart JK. Blepharokeratoconjunctivitis in children: diagnosis and treatment. Br J Ophthalmol. 2005;89(4): 400-403.

3. Wong IB, Nischal KK. Managing a child with an external ocular disease. J AAPOS. 2010;14(1):68-77.

4. Rubin M, Rao SN. Efficacy of topical cyclosporin $0.05 \%$ in the treatment of posterior blepharitis. J Ocul Pharmacol Ther. 2006;22(1):47-53.

5. Tatlipinar S, Akpek EK. Topical ciclosporin in the treatment of ocular surface disorders. Br J Ophthalmol. 2005;89(10):1363-1367.

6. Kunert KS, Tisdale AS, Stern ME, Smith JA, Gipson IK. Analysis of topical cyclosporine treatment of patients with dry eye syndrome: effect on conjunctival lymphocytes. Arch Ophthalmol. 2000;118(11): 1489-1496.

7. Fukushima A, Ozaki A, Fukata K, Ishida W, Ueno H. Ag-specific recognition, activation, and effector function of $\mathrm{T}$ cells in the conjunctiva with experimental immune-mediated blepharoconjunctivitis. Invest Ophthalmol Vis Sci. 2003;44(10):4366-4374.

8. Seal D, Ficker L, Ramakrishnan M, Wright P. Role of staphylococcal toxin production in blepharitis. Ophthalmology. 1990;97(12):1684-1688.

9. Tang-Liu DD, Acheampong A. Ocular pharmacokinetics and safety of ciclosporin, a novel topical treatment for dry eye. Clin Pharmacokinet. 2005;44(3):247-261.

10. Hasanreisoglu M, Avisar R. Long-term topical cyclosporin A therapy in Thygeson's superficial punctuate keratitis: a case report. Cases $J$. 2008;1:415.

11. Perry HD, Doshi-Carnevale S, Donnenfeld ED, Kornstein HS. Topical cyclosporine A $0.5 \%$ as a possible new treatment for superior limbic keratoconjunctivitis. Ophthalmology. 2003;110(8):1578-1581.

12. Perry HD, Doshi-Carnevale S, Donnenfeld ED, Solomon R, Biser SA, Bloom AH. Efficacy of commercially available topical cyclosporine A $0.05 \%$ in the treatment of meibomian gland dysfunction. Cornea. 2006;25(2):171-175.

13. Nelson JD, Shimazaki J, Benitez-del-Castillo JM, et al. The international workshop on meibomian gland dysfunction: report of the definition and classification subcommittee. Invest Ophthalmol Vis Sci. 2011;52(4):1930-1937.

14. Gupta N, Dhawan A, Beri S, D'souza P. Clinical spectrum of pediatric blepharokeratoconjunctivitis. JAAPOS. 2010;14(6):527-529.

15. Carnahan MC, Goldstein DA. Ocular complications of topical, peri-ocular, and systemic corticosteroids. Curr Opin Ophthalmol. 2000;11(6):478-483. 
International Medical Case Reports Journal

Dovepress

\section{Publish your work in this journal}

The International Medical Case Reports Journal is an international, peer-reviewed open-access journal publishing original case reports from all medical specialties. Previously unpublished medical posters are also accepted relating to any area of clinical or preclinical science. Submissions should not normally exceed 2,000 words or

4 published pages including figures, diagrams and references. The manuscript management system is completely online and includes a very quick and fair peer-review system, which is all easy to use. Visit $\mathrm{http}: / / \mathrm{www}$.dovepress.com/testimonials.php to read real quotes from published authors.

Submit your manuscript here: http://www.dovepress.com/international-medical-case-reports-journal-journal 